\title{
Budget impact analysis of niraparib for first-line maintenance therapy in advanced ovarian cancer from a US payer perspective
}

\author{
Jinan Liu, MD, PhD; Carol Hawkes, PhD; Lydia Walder, MSci; Chloe Spalding, PhD; Karin Travers, DSci; \\ Eric M Maiese, PhD, MHS; and Jean Hurteau, MD, PhD
}

\section{What is already known about this subject}

- Despite the aggressive first-line treatment of patients with advanced epithelial ovarian cancer with surgical cytoreduction and systemic platinumbased chemotherapy, up to $85 \%$ of patients will experience a recurrence.

- In the phase III randomized doubleblind PRIMA (PRIMA/ENGOT-OV26/ GOG-3012) trial, niraparib maintenance treatment demonstrated a clinically meaningful improvement of progression-free survival compared with the placebo.

- Niraparib was recently approved by the United States (US) FDA for the maintenance treatment of adult patients with advanced epithelial ovarian cancer $(\mathrm{OC})$ who are in complete or partial response to firstline platinum-based chemotherapy regardless of biomarker status, and the approval is expected to result in niraparib's market share increasing as a first-line maintenance therapy.

\section{ABSTRACT}

BACKGROUND: Ovarian cancer (OC) is the fifth leading cause of cancer death in women and has the highest mortality rate of gynecological cancers. Niraparib was recently approved by the FDA for the maintenance treatment of adult patients with advanced epithelial OC in complete or partial response to first-line platinum-based chemotherapy (PBC) regardless of biomarker status.

\author{
What this study adds \\ - This budget impact model (BIM) \\ demonstrates a minimal economic \\ impact of adding niraparib as an \\ approved first-line maintenance \\ treatment for patients with advanced \\ epithelial OC after a complete or \\ partial response to platinum-based \\ chemotherapy. \\ - Treatment acquisition cost was the \\ main driver of the budget impact of \\ niraparib as a first-line maintenance \\ treatment for patients with advanced \\ epithelial OC.
}

\author{
Author affiliations \\ Jinan Liu, MD, PhD; Karin Travers, DSci; and \\ Jean Hurteau, MD, PhD, GlaxoSmithKline, \\ Waltham, MA. Carol Hawkes, PhD, \\ GlaxoSmithKline, Uxbridge, UK. Lydia \\ Walder, MSci, and Chloe Spalding, PhD, \\ FIECON, London, UK. Eric M Maiese, PhD, \\ MHS, GlaxoSmithKline, Philadelphia, PA. \\ AUTHOR CORRESPONDENCE: \\ Jinan Liu, 339.970.0900; jinan.x.liu@gsk.com
}

\author{
$J$ Manag Care Spec Pharm. \\ 2021;27(10):1337-87 \\ Copyright $\odot 2021$, Academy of Managed \\ Care Pharmacy. All rights reserved.
}

OBJECTIVE: To estimate the direct economic impact on US payers of adding niraparib as a first-line maintenance therapy for patients with advanced OC.

METHODS: The model considered 2 scenarios: a current scenario in which niraparib does not have regulatory approval for first-line maintenance therapy and a future scenario in which niraparib has regulatory approval for first-line maintenance therapy. The budget impact was calculated as the difference in cost between the 2 scenarios. The budget impact model (BIM) considered 2 different US health care payer perspectives: a commercial health plan and a Medicare plan. Both payer perspectives were assumed to have a hypothetical 1 million affiliates that were covered. Epidemiological data was used to estimate the eligible incident population of patients with OC. Active surveillance, bevacizumab (as a monotherapy), and olaparib (as a monotherapy restricted to patients with the breast 
cancer gene $[B R C A]$ mutation) were included in the model as alternative maintenance treatment options (maintenance treatment options required $1 \%$ market share for inclusion). Cost categories considered in the BIM included diagnostic testing, treatment acquisition and administration, treatment-emergent adverse events, and subsequent therapy. Results were presented as an incremental budget impact to payers over 3 years.

RESULTS: For a commercial health plan of 1 million affiliates, the estimated impact of adding niraparib as a first-line maintenance treatment option for advanced epithelial $O C$ was calculated as $\$ 87,906, \$ 93,106$, and $\$ 87,037$ for years 1,2 , and 3 , respectively. The average budget impact per member per month was $\$ 0.007$. For a Medicare health plan of 1 million affiliates, the estimated impact was calculated as $\$ 206,785, \$ 219,017$, and $\$ 204,739$ for years 1,2 , and 3, respectively. The average budget impact per member per month was $\$ 0.018$. One-way sensitivity analyses suggested that budget impact was most sensitive to the treatment duration and market share of niraparib, the non-treatment-specific data on overall survival rates, and the treatment duration of bevacizumab. Treatment of drug-specific adverse events had little impact on the budget model.

CONCLUSIONS: The model estimated a minimal budget impact to both a commercial or Medicare health plan following the introduction of niraparib as a first-line maintenance therapy for patients with advanced epithelial $\mathrm{OC}$ who are in complete or partial response to first-line PBC regardless of biomarker status.

Ovarian cancer (OC) accounts for approximately $2.5 \%$ of all women's cancers in the United States. ${ }^{1-3}$ Approximately 21,750 new cases of OC were expected in 2020, which will make OC the 11th most common cancer among women., ${ }^{2,3}$ OC is typically diagnosed later in life with a median age of 63 years at diagnosis. ${ }^{2}$ Although relatively rare, OC would be the fifth leading cause of cancer death in women in 2020 with an estimated 13,940 deaths; OC has the highest mortality rate of all gynecological cancers. ${ }^{2,3}$ The discrepancy in the case to mortality rate is often attributed to a lack of specific early symptoms leading to disease diagnosis in more advanced stages, the absence of screening methods for early diagnosis and treatment where survival is improved, and a lack of durable responses to first-line interventions. ${ }^{4}$ Nearly $70 \%$ of all cases are diagnosed in the advanced stages and have a poor prognosis with a 5 -year survival rate of $48 \%{ }^{1,4}$ Standard first-line treatment for advanced epithelial OC is surgical cytoreduction and systemic platinum-based chemotherapy (PBC). Unfortunately, despite these aggressive treatment modalities, up to $85 \%$ of patients with advanced OC will experience a recurrence. ${ }^{4}$

The US Food and Drug Administration (FDA) approved niraparib in 2020 for the maintenance treatment of adult patients with advanced epithelial ovarian, fallopian tube, or primary peritoneal cancer who are in complete or partial response to first-line PBC..$^{5}$ Niraparib is an oral, highly selective poly (adenosine diphosphate-ribose) PARP1 and PARP2 inhibitor. ${ }^{6}$ It is the first PARP inhibitor to show clinical efficacy as a maintenance monotherapy for patients with advanced epithelial OC in the first-line setting, as well as in the platinum-sensitive recurrent setting, regardless of biomarker status. In the PRIMA (PRIMA/ ENGOT-OV26/GOG-3012) trial, in the intention-to-treat population, patients with advanced $\mathrm{OC}$, regardless of homologous-recombination-deficiency status, who received niraparib after having a response to first-line PBC had longer progression-free survival (PFS) than those receiving the placebo (13.8 months vs 8.2 months; hazard ratio for disease progression or death, $0.62 ; 95 \% \mathrm{CI}=0.50-0.76$, $\mathrm{P}<0.001){ }^{6}$ These results are consistent with the NOVA trial, which led to the FDA approval of niraparib in 2017 for maintenance treatment in patients with platinum-sensitive recurrent $\mathrm{OC}$ who were in complete or partial response to PBC regardless of biomarker status. ${ }^{7}$ In the germline breast cancer gene (BRCA) mutation cohort (gBRCAm) of the NOVA trial, patients treated with niraparib had a significantly longer median duration of PFS than patients receiving placebo (hazard ratio $=0.27,95 \% \mathrm{CI}=0.17-0.41$ ). In the overall non-gBRCAm cohort, patients receiving niraparib also had a significantly longer median duration of PFS than patients receiving placebo (hazard ratio $=0.45,95 \% \mathrm{CI}=0.34-0.61) .{ }^{8}$

Herein we present a budget impact model (BIM) that was developed to estimate the economic impact of adding niraparib as a first-line maintenance therapy for patients with advanced $\mathrm{OC}$ who are in complete or partial response to first-line PBC from a payer perspective in the United States.

\section{Methods}

The BIM was developed in Microsoft Excel (Excel Version 2002; Redmond, WA) according to The Professional Society for Health Economics and Outcomes Research (ISPOR) guidelines. ${ }^{9}$ The model considered 2 scenarios: a current scenario in which niraparib did not have first-line maintenance regulatory approval and a future scenario in which niraparib had regulatory approval for first-line maintenance therapy. The incremental budget impact was calculated as the difference in costs between these 2 scenarios. Results of these analyses were presented as an incremental budget impact to payers over 3 years in the United States. A framework for the BIM is shown in Figure 1. 


\section{FIGURE 1 Overview of the BIM Framework}

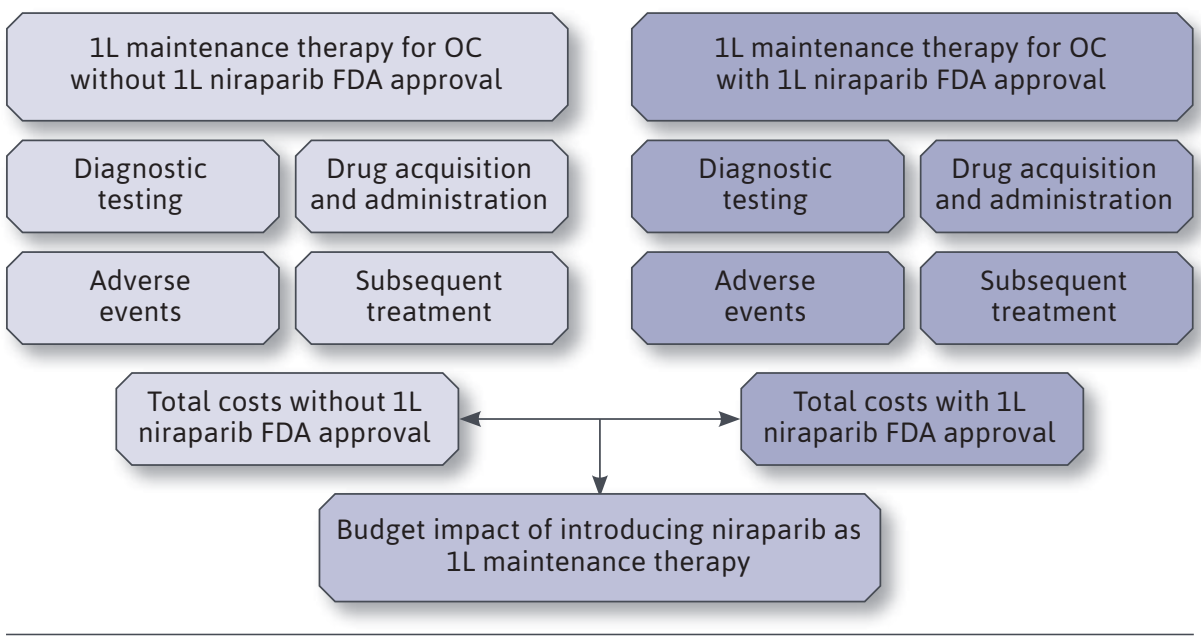

$1 \mathrm{~L}=$ first-line; $F D A=$ Food and Drug Administration; $O C=$ ovarian cancer and administration, adverse events, and subsequent treatment (Figure 1). In addition, the incremental health plan costs resulting from the availability of niraparib were presented in a range of relevant outputs like, for example, the annual budget impact per member per month in dollars. Deterministic sensitivity analyses, which assess how sensitive a model is to any given variable, were performed to explore the level of uncertainty in the model results.

\section{NUMBER OF ELIGIBLE PATIENTS}

The BIM was developed to consider a base case that was aligned with the US payer perspective and the indicated regulatory approval of niraparib as a maintenance treatment in the first-line setting. Eligible patients included those patients newly diagnosed with high-grade epithelial (serous and endometrioid) ovarian, peritoneal, or fallopian tube cancer, following response (either complete or partial) to first-line PBC. ${ }^{6}$ To calculate the incident population eligible for treatment with niraparib in year 1 , the following determinants were used (Supplementary Figure 1; Supplementary Table 1, available in online article).

- Total: An initial population of 1 million affiliates was assumed for the perspective of a health plan in the US.

- Age groups: The initial population of affiliates was categorized by age group (one group from 19 to 25 years, one group from 26 to 34 years, and then grouped into 10 -year blocks until the final group of at least 65 years). US health insurance status data were used to determine the proportion of affiliates in each category for either the commercial or Medicare plan..$^{15,16}$

- Female: The number of female patients was determined for each for use as a monotherapy (restricted to for use as a monotherapy, and nirapaib pre-FDA approval utilization. ${ }^{11-13}$ approved as a first-line maintenance until April 2020, market share data indicated that there was utilization as a first-line maintenance treatment share data showed more than $1 \%$ of the market utilization in this patien criteria for inclusion in the BIM as an contion.

the direct economic impact to payers and was therefore dependent on the estimated number of patients and the costs associated with their treatment. To determine costs, clinical inputs, inputs were applied (Figure 1).

In the BIM, patients received either niraparib or an alternative treatment option according to the market share data. ${ }^{14}$ Annual costs for each treatment were estimated and included the costs for diagnostic testing, drug acquisition the following treatment options were included: active surveillance, olaparib 
age category using population data that had been categorized by age and sex. ${ }^{15}$

- OC incidence: US age-specific incidence rates were applied to determine the number of female affiliates with OC in each group. The total number of patients with OC was then calculated by determining the sum of the affiliates with $\mathrm{OC}$ in each age group. ${ }^{17}$

- Advanced stage: The proportion of advanced OC patients (International Federation of Gynecology and Obstetrics stage III or IV; $70 \%$ ) was applied to the total number of female affiliates with OC. ${ }^{4}$

- Eligible for first-line PBC: The proportion of patients eligible for first-line PBC $(100 \%)$ was then applied to the total number of patients with advanced OC. This proportion assumes that all patients diagnosed with advanced OC were initially considered for first-line PBC.

- Response to first-line treatment: The base case overall population was then obtained by applying the proportion of patients who respond to treatment (collapsed for complete/partial response; $75 \%$ ) to the proportion of patients who received first-line PBC..$^{18}$

An overview of the estimated number of incident patients eligible for treatment with niraparib as a first-line maintenance therapy for a commercial health plan and Medicare health plan is shown in Supplementary Figures 2 and $\underline{3}$, respectively, available in online article. (Greater detail on the breakdown of the data can be found in Supplementary Tables 14 and $\underline{15}$, respectively, available in online article.) The eligible population was assumed to increase each year in line with the population growth in the United States (for the model, a growth rate of $0.71 \%$ was used)..$^{19}$ When patients remained on treatment for longer than 1 year, mortality rates were applied to account for the number of patients eligible for treatment. OC-specific overall survival (OS) data were applied to the patient cohort, as this was expected to be higher than the overall mortality rates in the general population. In the absence of mature treatmentspecific OS rates, a conservative approach was taken in which mature non-treatment-specific OS data were used in the model for all treatments (Supplementary Table 2) ${ }^{20}$ An example of how OS influenced the 3-year time horizon was provided for the commercial health plan (Supplementary Table 3 , available in online version).

\section{UPTAKE ASSUMPTIONS}

Market share data within the current scenario were obtained from the nationwide Flatiron Health electronic-health-record-derived de-identified database and were supplemented with market research conducted by GlaxoSmithKline and expert clinical input. ${ }^{21}$ The Flatiron
Health database is a longitudinal database comprising de-identified patient-level structured and unstructured data and is curated via technology-enabled abstraction, which includes approximately 280 cancer clinics (roughly 800 sites of care). The majority of patients in the database originate from community oncology settings; relative community/academic proportions may vary depending on the study cohort. To determine the market uptake of niraparib following regulatory approval, user-defined market share capture rates were used. The capture rates defined the proportion of patients taken from each treatment option. Market research was conducted, and expert clinical opinions were sought to predict the anticipated market uptake for niraparib in the future scenario (Supplementary Table 4 , available in online version). Market share data would include the gBRCAm limitation for olaparib use. In addition, after approval, niraparib would also capture $100 \%$ of the preapproval market share of niraparib.

\section{DIAGNOSTIC TESTING}

A germline BRCA diagnostic test is recommended in all patients with epithelial OC in the United States to screen patients and families for hereditary causes of breast and OC. As such, BRCA diagnostic testing is considered routine care within the United States, and all patients entering the BIM underwent a BRCA diagnostic test. ${ }^{22,23}$ The cost of a germline BRCA diagnostic test for a commercial health plan was estimated at $\$ 8,184$, whereas the cost was estimated at $\$ 5,591$ for a Medicare health plan. Costs were sourced from published literature and inflated to a 2018 cost-year using US inflation indices available at the time the model was initially developed. ${ }^{24}$

\section{DRUG ACQUISITION AND ADMINISTRATION COSTS}

Treatment costs were applied for all patients receiving a maintenance treatment until the time that a patient discontinued treatment. The model considered time on treatment using results from the key primary clinical trials for each treatment option. Clinical expert opinion indicated that the restricted mean time estimated PFS from the area under the Kaplan-Meier curves were the most appropriate endpoint for modeling time on maintenance treatment.

Stopping rules for treatments were applied according to marketing regulations in the United States. Patients within the active surveillance arm did not receive any active treatment and, therefore, had no stopping rule applied. Since the time horizon of the BIM was 3 years, no stopping rule was applied for niraparib (Supplementary Table 5, available in online article). Bevacizumab had a stopping rule of 22 cycles (21 days per cycle) as per the FDA label. Therefore, a stopping rule of 1.27 years was applied for bevacizumab in 


\section{TABLE 1 Overview of Budget Impact Results for the Base Case Settings for a Commercial Health Plan}

\begin{tabular}{|c|c|c|c|c|c|}
\hline Category & 2020 & 2021 & 2022 & $\begin{array}{c}\text { Average over } \\
3 \text { years }\end{array}$ & $\begin{array}{c}\text { Total over } 3 \\
\text { years }\end{array}$ \\
\hline Patients eligible for treatment with niraparib & 46 & 88 & 123 & 86 & 257 \\
\hline Patients treated with niraparib & 2 & 7 & 13 & 7 & 22 \\
\hline Total costs in scenario without niraparib regulatory approval, \$ & $3,715,648$ & $5,056,271$ & $5,471,354$ & $4,747,758$ & $14,243,273$ \\
\hline Total costs in scenario with niraparib regulatory approval, \$ & $3,803,554$ & $5,149,377$ & $5,558,390$ & $4,837,107$ & $14,511,321$ \\
\hline Annual budget impact, \$ & 87,906 & 93,106 & 87,037 & 89,350 & 268,049 \\
\hline Budget impact, \% & 2.37 & 1.84 & 1.59 & 1.88 & 1.88 \\
\hline Annual budget impact per patient treated with niraparib, \$ & 38,285 & 13,820 & 6,731 & 12,204 & 12,204 \\
\hline Annual budget impact per patient treated with niraparib per month, $\$$ & 3,190 & 1,152 & 561 & 1,017 & 1,017 \\
\hline Annual budget impact per member, $\$$ & 0.088 & 0.093 & 0.087 & 0.089 & 0.268 \\
\hline Annual budget impact per member per month, $\$$ & 0.007 & 0.008 & 0.007 & 0.007 & 0.022 \\
\hline
\end{tabular}

the BIM. ${ }^{25}$ As per the SOLO-1 trial, olaparib had a stopping rule of 2 years (Supplementary Table 5$){ }^{26}$

Drug dosage and costs used in the BIM are reported in Supplementary Table 6, available in online article. Daily dosing for niraparib and olaparib were informed by the dose intensity recorded in the PRIMA trial. Because of an absence of published relative dose intensity data from SOLO-1, the relative dose intensity from PRIMA (87\%) was also applied to the fixed dose of olaparib used in the SOLO-1 trial. The dose of bevacizumab was based on the FDA label. ${ }^{25}$ Wholesale acquisition costs (WAC) for all active treatments were obtained from the Wolters Kluwer database (New York, NY). ${ }^{27}$ It was assumed that oral therapies did not incur any administration costs. As bevacizumab is administered intravenously, a \$143 administration cost was applied per treatment administration. ${ }^{28}$ This administration cost was determined as 1 hour of intravenous (IV) administration and was sourced from the Medicare coding and payment schedule. $^{29}$

Treatment costs were applied for the time on treatment, which was equal to the shorter of the chosen endpoint or the stopping rule. Treatment duration based on mean PFS and discontinuation rules are presented in Supplementary Table 7, available in online article.

Adverse Events. The BIM included costs that were associated with a grade of at least 3 treatment-related adverse events (TRAEs) that were reported in at least $5 \%$ of patients in the primary clinical trials for all treatment options. ${ }^{6,26,30}$ It was expected that TRAEs would occur when a patient initiated treatment and that the TRAE would be managed by dose modification and interruptions where applicable. Therefore, associated costs were applied as a one-time cost in the first year of treatment (Supplementary Table 8 , available in online version). TRAE costs were sourced from published literature..$^{28}$ The cost for fatigue was assumed to be equal to 1 outpatient visit, whereas the cost for leukopenia was assumed to be equal to neutropenia.

Subsequent Treatment. Second-line subsequent treatment costs were applied to all patients when they discontinued primary treatment (Supplementary Table 9, available in online version). Data used to inform subsequent therapy were sourced from the PRIMA trial and US clinical expert opinion. ${ }^{31}$ Data for olaparib and bevacizumab subsequent treatment were not publicly available, so they were assumed to be equivalent to niraparib. WAC costs for subsequent treatments were sourced from the Wolters Kluwer database. ${ }^{27} \mathrm{~A}$ total cost per class of treatment was calculated based on the average treatment duration sourced from published literature and WAC costs..$^{27,32}$

Number of Patients on Niraparib Treatment. To calculate the number of patients on niraparib for the BIM, market uptake values were applied to the eligible population. The niraparib market share was assumed to be $5 \%, 10 \%$, and $15 \%$ in years 1, 2, and 3, respectively. Supplementary Table 10 and Supplementary Figure 4, available in online article, describe the estimated number of patients receiving niraparib in the commercial health plan over the 3-year time horizon. Supplementary Table 11 and Supplementary Figure 5, available in online article, present the same information for a Medicare plan scenario. 


\begin{tabular}{|c|c|c|c|c|c|}
\hline Category & 2020 & 2021 & 2022 & $\begin{array}{c}\text { Average } \\
\text { over } 3 \text { years }\end{array}$ & $\begin{array}{c}\text { Total } \\
\text { over } 3 \text { years }\end{array}$ \\
\hline Patients eligible for treatment with niraparib & 108 & 208 & 289 & 202 & 605 \\
\hline Patients treated with niraparib & 5 & 16 & 30 & 17 & 52 \\
\hline Total costs in scenario without niraparib regulatory approval, \$ & $8,460,361$ & $11,611,969$ & $12,586,381$ & $10,886,237$ & $32,658,712$ \\
\hline Total costs in scenario with niraparib regulatory approval, \$ & $8,667,146$ & $11,830,986$ & $12,791,120$ & $11,096,418$ & $33,289,253$ \\
\hline Annual budget impact, \$ & 206,785 & 219,017 & 204,739 & 210,180 & 630,541 \\
\hline Budget impact, \% & 2.44 & 1.89 & 1.63 & 1.93 & 1.93 \\
\hline Annual budget impact per patient treated with niraparib, $\$$ & 38,285 & 13,820 & 6,731 & 12,204 & 12,204 \\
\hline Annual budget impact per patient treated with niraparib per month, $\$$ & 3,190 & 1,152 & 561 & 1,017 & 1,017 \\
\hline Annual budget impact per member, $\$$ & 0.207 & 0.219 & 0.205 & 0.210 & 0.631 \\
\hline Annual budget impact per member per month, $\$$ & 0.017 & 0.018 & 0.017 & 0.018 & 0.053 \\
\hline
\end{tabular}

\section{Results}

\section{BASE CASE FOR A COMMERCIAL HEALTH PLAN}

The base case for the commercial health plan perspective estimated the budget impact of adding niraparib as a firstline treatment for patients with $\mathrm{OC}$ who have responded to first-line PBC. For a commercial health plan with 1 million affiliates, the model estimates that 2 patients will be treated with niraparib in year 1 , which increased to 7 and 13 patients in years 2 and 3, respectively. The overall budget impact of adding niraparib was calculated as $\$ 87,906, \$ 93,106$, and $\$ 87,037$ for years 1, 2, and 3, respectively (Table 1). For a plan with 1 million affiliates, the budget impact, when averaged over the 3-year time horizon, amounted to an average budget impact per patient treated with niraparib per month of $\$ 1,017$, which corresponded to an average budget impact per member per month of \$0.007 (Table 1).

Treatment acquisition and administration costs, as well as costs associated with TRAEs, were estimated to be higher in years 1, 2, and 3 of the future scenario compared to the current scenario (Supplementary Table 12, available in online article). However, because fewer patients in the niraparib arm of the PRIMA trial received subsequent therapy and there is an increase in patients receiving niraparib instead of an alternative option in the future scenario, subsequent therapy costs decreased in the future scenario (Supplementary Table 12).

\section{BASE CASE FOR A MEDICARE PLAN}

For a Medicare plan with 1 million affiliates, the model estimates that 5 patients will be treated with niraparib in year 1 , which increased to 16 and 30 patients in years 2 and 3, respectively. An additional 30 patients are expected to receive niraparib on the Medicare plan when compared to the commercial plan because of a higher OC incidence rate for women in the 65+ age category. The overall budget impact of adding niraparib was calculated as $\$ 206,785, \$ 219,017$, and $\$ 204,739$ for years 1, 2, and 3, respectively (Table 2). For a plan with 1 million affiliates, the budget impact, when averaged over the 3-year time horizon, amounted to an average budget impact per patient treated with niraparib per month of $\$ 1,017$, which corresponded to an average budget impact per member per month of \$0.018 (Table 2).

Treatment acquisition and administration costs, as well as costs associated with TRAEs, were estimated to be higher in years 1, 2, and 3 of the future scenarios than in the current scenario (Supplementary Table 13, available in online version). However, because fewer patients in the niraparib arm of the PRIMA trial received subsequent therapy and there is an increase in patients receiving niraparib, instead of an alternative option in the future scenario, subsequent therapy costs decreased in the future scenario (Supplementary Table 13).

\section{SENSITIVITY ANALYSES}

A one-way sensitivity analysis (OWSA) considers how sensitive a BIM is to changes in any parameter value. An OWSA uses confidence intervals sourced from prespecified probabilistic distributions. ${ }^{28}$ For this BIM, the base case for all parameters was assumed to have a standard error of $20 \%$ of the mean value to examine the sensitivity of results. ${ }^{33}$ Upper and lower bounds were calculated according to distributions that are fitted. The budget impact was calculated using the lower and upper bounds for each parameter value, 


\section{FIGURE 2 One-Way Sensitivity Analysis of the Budget Impact Per Member Per Month Averaged Over 3 Years} for a Commercial Health Plan

Result from lower bound

Result from upper bound

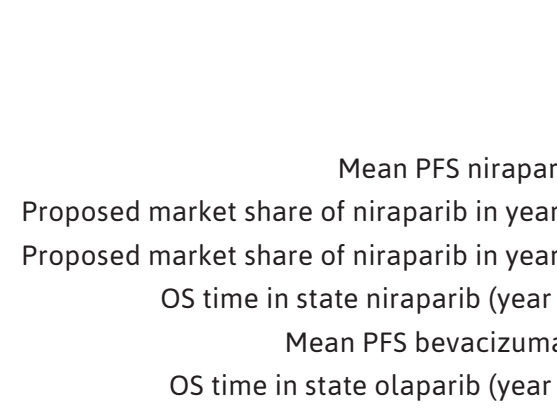

Proposed market share of niraparib in year 1 Patients with MA status (N.B. proportion of OC patients within niraparib's license) Incident number of patients eligible for treatment with niraparib Subsequent chemotherapy costs for niraparib OS time in state niraparib (pre-FDA approval; year 1) Subsequent chemotherapy costs for bevacizumab OS time in state active surveillance (year 1 ) Subsequent chemotherapy costs for active surveillance Mean PFS olaparib Subsequent chemotherapy costs for niraparib (pre-FDA approval) Mean PFS active surveillance OS time in state bevacizumab (year 1) Subsequent chemotherapy costs for olaparib OS time in state olaparib (year 2)
Annual budget impact per member per month (\$) average over 3 years

\begin{tabular}{ccccc}
-0.01 & 0.00 & 0.01 & 0.02 & 0.03 \\
\hline
\end{tabular}

FDA = Food and Drug Administration; OS=overall survival; $P F S=$ progression-free survival

and a tornado diagram was used to display the 20 most sensitive parameters with the most sensitive parameters displayed along the $y$-axis. The sensitivity of a parameter is described by the size of the difference between the budget impact values associated with the lower and upper bounds.

The OWSA estimated that the budget impact per member per month following the introduction of niraparib, averaged over 3 years, was below $\$ 0.022$ for all parameter variations for the commercial health plan and was below $\$ 0.051$ for the Medicare plan. The results were most sensitive to the treatment duration of niraparib (PFS), market share of niraparib, OS rate of niraparib, treatment duration of bevacizumab, and OS rate of olaparib (Figure 2 and Figure 3).

\section{Discussion}

This analysis was undertaken to estimate the budget impact resulting from the FDA approval of niraparib for the first-line maintenance treatment of patients with advanced epithelial OC who had a complete or partial response to PBC regardless of biomarker status. The population of eligible patients in this BIM is small and well defined with a limited number of available maintenance treatments. In a base case analysis, for a 1 million hypothetical patient population on a commercial health plan, over the 3-year time horizon the average budget impact per patient treated with niraparib per month was estimated as $\$ 1,017$, which equated to an average budget impact per member per month of $\$ 0.007$. For a 1 million patient population on a Medicare plan, the average budget impact per patient treated with niraparib per month and per member per month were $\$ 1,017$ and $\$ 0.018$, respectively, over 3 years.

In the BIM, 2 scenarios were considered: a current scenario in which niraparib did not have first-line regulatory approval and a future scenario in which niraparib did have first-line regulatory approval. The analysis examined 


\section{FIGURE 3 One-Way Sensitivity Analysis of the Budget Impact Per Member Per Month Averaged Over 3 Years} for a Medicare Plan

Result from lower bound

Result from upper bound

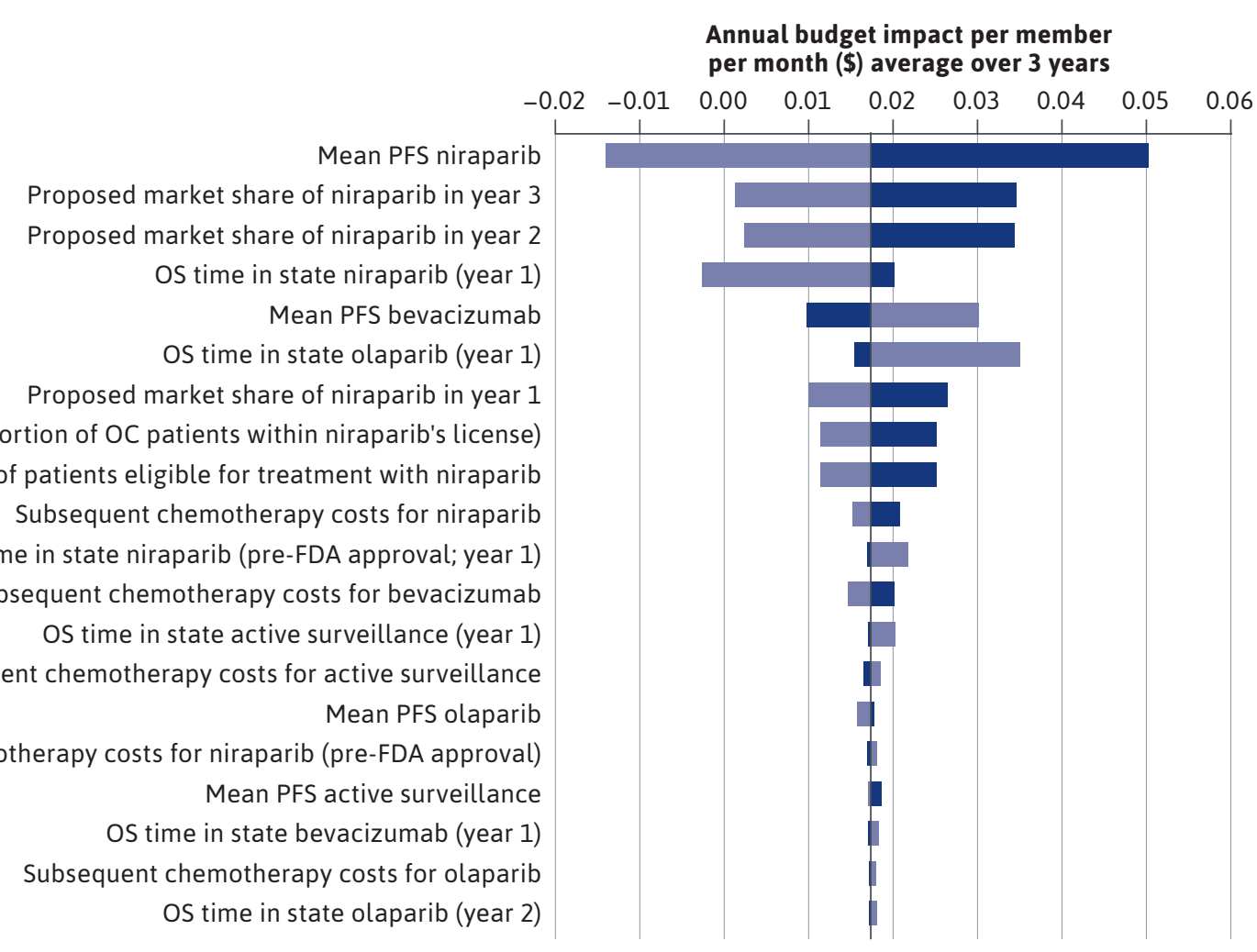

$F D A=$ Food and Drug Administration; $O S=$ overall survival; $P F S=$ progression-free survival

the cost of alternate treatment regimens, the cost for treating drug-specific TRAEs, and the cost of subsequent treatment in patients who discontinued treatment over a 3-year period. Alternative maintenance treatment options included active surveillance, olaparib monotherapy, and bevacizumab monotherapy. The analysis did not include an olaparib/bevacizumab combination maintenance therapy, such as the one used in the PAOLO- 1 trial, because the combination therapy did not reach the $1 \%$ market share necessary to be included in the BIM at the time of this analysis. The OWSA suggested that the budget impact was most sensitive to the treatment duration of niraparib (PFS), market share of niraparib, OS rates of both niraparib and olaparib, and the treatment duration of bevacizumab. However, since non-treatment-specific OS data were used, the model may potentially underestimate costs for treatments with higher OS rates.

\section{LIMITATIONS}

The majority of the limitations of this BIM occur because of limited data availability and structural uncertainty. Because of a lack of mature OS data, OS is assumed to be the same for each treatment option, leading to an assumption of equal mortality across treatments. Therefore, the BIM may underestimate the number of patients on treatments with high OS rates, leading to an underestimation of the costs associated with those treatments with better survival.

Two additional limitations of the BIM could potentially lead to an underestimation of costs. First, the BIM only considered 2 payer perspectives, either a commercial health plan or a Medicare plan, which limits the findings to a representation of this population only and is not for an overall US population model. Second, the model base case considered incident cases only. This assumption leads to a potential underestimation of the number of patients eligible for treatment and, thus, an underestimation of 
costs. However, prevalent patients usually receive treatment shortly after diagnosis, and, therefore, few would be eligible for first-line maintenance therapy following $\mathrm{PBC}$. Conversely, within the BIM, time on treatment was determined by PFS data and was used to determine the time for which treatment costs were applied. Alternative reasons for treatment discontinuation were not considered, and, therefore, treatment acquisition costs could possibly be overestimated in the BIM. Furthermore, because data for olaparib and bevacizumab subsequent treatment were not publicly available, the BIM assumed that the subsequent treatment costs were equivalent to those for niraparib. This may lead to either an overestimation or an underestimation of subsequent treatment costs.

Other limitations of the BIM arise from the use of olaparib. The average dose intensity recorded in the SOLO-1 trial was not publicly available, and therefore, the BIM assumed that patients on olaparib received a dose that was aligned to the relative dose intensity of niraparib in the PRIMA trial. This assumption may have led to an underestimation of olaparib treatment costs in the BIM. Furthermore, per the SOLO-1 trial protocol, the BIM assumed a maximum olaparib treatment time of 2 years. However, physicians may decide to continue treatment with olaparib if patients demonstrate a partial response, and some patients may continue treatment beyond 2 years. Therefore, the drug acquisition costs for olaparib may be underestimated.

Despite the limitations of the analysis, there are a number of strengths that should be noted. The BIM considered the changes in treatment-related costs attributable to the changes in eligible population over the time course of the model, which is a strength of this analysis. This analysis included the impact of niraparib, olaparib, and bevacizumab efficacy, which could influence the size of the eligible population. In addition, the model included all the treatment-related acquisition, administration, and adverse event costs. The main driver of overall budget impact for both the commercial and Medicare health plans was treatment acquisition costs. This finding is in line with other budget impact models of maintenance treatments for $\mathrm{OC}$, which demonstrates that the variable with the most significant impact on the model is the price of drug acquisition..$^{34,36}$

\section{Conclusions}

The model estimated a minimal budget impact to either a commercial or Medicare health plan following the introduction of niraparib as a firstline maintenance therapy of patients with advanced epithelial OC who are in complete or partial response to $\mathrm{PBC}$ regardless of biomarker status.

\section{DISCLOSURES}

This study was financially supported by GlaxoSmithKline. Liu, Hawkes, Maiese, and Hurteau are employees of GlaxoSmithKline. Travers was employed by GlaxoSmithKline at the time of this study. Spalding and Walder are employees of FIECON Ltd., which was contracted by GlaxoSmithKline to develop the budget impact model used in this study.

\section{REFERENCES}

1. American Society of Clinical Oncology. Ovarian, fallopian tube, and peritoneal cancer: statistics. Cancer.Net. Published May 2020. Accessed October 5, 2020. https://www. cancer.net/cancer-types/ovarianfallopian-tube-and-peritoneal-cancer/ $\underline{\text { statistics }}$
2. American Cancer Society. Key statistics for ovarian cancer. American Cancer Society. Accessed October 5, 2020. https://www.cancer.org/cancer/ovariancancer/about/key-statistics.html

3. Ovarian Cancer Research Alliance. Statistics. OCRAHope. Accessed October 5, 2020. https://ocrahope.org/patients/ about-ovarian-cancer/statistics/

4. Cortez AJ, Tudrej P, Kujawa KA, Lisowska KM. Advances in ovarian cancer therapy. Cancer Chemother Pharmacol. 2018;81(1):17-38. doi:10.1007/ s00280-017-3501-8

\section{ZEJULA. Prescribing informa-} tion. GlaxoSmithKline; 2020. Accessed October 6, 2020. https://www. accessdata.fda.gov/drugsatfda docs/ label/2020/208447s015s017lbledt.pdf

6. González-Martín A, Pothuri B, Vergote I, et al. Niraparib in patients with newly diagnosed advanced ovarian cancer. N Eng J Med. 2019;381:2391-402. doi:10.1056/NEJMoa1910962

7. US Food and Drug Administration. Niraparib (ZEJULA). US Food and Drug Administration. Published May 30, 2017. Accessed November 11, 2020. https://www.fda.gov/drugs/ resources-information-approved-drugs / niraparib-zejula

8. Mirza M, Monk B, Herrstedt J, et al. Niraparib maintenance therapy in platinum-sensitive, recurrent ovarian cancer. N Eng J Med. 2016;375:2154-64. doi:10.1056/NEJMoa1611310

9. Sullivan SD, Mauskopf JA, Augustovski F, et al. Budget impact analysis-principles of good practice: report of the ISPOR 2012 Budget Impact Analysis Good Practice II Task Force. Value Health. 2014;17(1):5-14. doi:10.1016/j.jval.2013.08.2291

10. US Food and Drug Administration. FDA approves niraparib for first-line maintenance of advanced ovarian cancer. US Food and Drug Administration. Published April 29, 2020. Accessed October 6, 2020. https://www.fda.gov/ drugs/drug-approvals-and-databases/ fda-approves-niraparib-first-line-maintenance-advanced-ovarian-cancer 
11. European Medicines Agency. Lynparza: Olaparib. European Medicines Agency. Published October 6, 2020. Accessed October 6, 2020. https://www.ema. europa.eu/en/medicines/human/EPAR/ lynparza

12. US Food and Drug Administration. FDA approved olaparib (LYNPARZA, AstraZeneca Pharmaceuticals LP) for the maintenance treatment of adult patients with deleterious or suspected deleterious germline or somatic BRCA-mutated (gBRCAm or sBRCAm) advanced epithelial ovarian, fallopian tube or primary peritoneal cancer who are in complete or partial response to first-line platinum-based. US Food and Drug Administration. Published December 26, 2018. Accessed October 6, 2020. http://www.fda.gov/drugs/ fda-approved-olaparib-lynparza-astrazeneca-pharmaceuticals-lp-maintenancetreatment-adult-patients

13. US Food and Drug Administration. FDA approves bevacizumab in combination with chemotherapy for ovarian cancer. US Food and Drug Administration. Published July 13, 2018. Accessed June 17, 2019. http://www.fda.gov/drugs/ resources-information-approved-drugs / fda-approves-bevacizumab-combinationchemotherapy-ovarian-cancer

14. GlaxoSmithKline. Data on file: flatiron analysis for PRIMA US BIM comparators.

15. United States Census Bureau. Age and sex composition in the United States: 2018. Census. Accessed October 6, 2020. https://www.census.gov/data/ tables/2018/demo/age-and-sex/2018age-sex-composition.html

16. United States Census Bureau. H-01 type of health insurance coverage by selected characteristics: 2018. Census. Accessed October 6, 2020. https://www. census.gov/data/tables/time-series/ demo/income-poverty/cps-hi/hi.html
17. National Cancer Institute. Surveillance, Epidemiology, and End Results Program: ovarian cancer incidence rates by age at diagnosis. National Cancer Institute SEER. Accessed October 6, 2020. https:// seer.cancer.gov/explorer/application. php? site $=61 \&$ data type $=1 \&$ graph type $=3 \&$ compareBy $=$ race $\&$ hdn $\quad$ sex $=\&$ chk race_1=1\&chk_data_type_1=1\&advopt_ precision $=1 \&$ showDataFor $=$ data_type $\_1$

18. Kemp Z, Ledermann J. Update on first-line treatment of advanced ovarian carcinoma. Int J Womens Health. 2013;5:45-51. doi:10.2147/IJWH.S30231

19. World Bank. Population growth rate. Accessed March 2, 2020. https://www. google.com/publicdata/explore ?ds=d5bncppjof8f9_\&met_y=sp_pop_gro w\&idim=country:USA:IND:CAN\&hl=en \&dl=en\#!ctype $=1 \&$ strail $=$ false $\& b c s=d \& n$ selm $=h \&$ met $y=s p$ pop grow\&scale $\mathrm{y}=$ lin\&ind $\mathrm{y}=$ false\& $\mathrm{rdim}=$ region\&idim $=$ country:USA:IND:CAN\&ifdim=region\&hl =en_US\&dl=en\&ind=false

20. Westin SN, Louie-Gao M, Badamgarav E, Bala MV, Thaker PH. Risk factors for progression or death in ovarian cancer patients who completed firstline platinum treatment. J Clin Oncol. 2019;37(Suppl 15):5548. doi:10.1200/ JCO.2019.37.15_suppl.5548

21. Flatiron. Flatiron Health EHRderived database. Flatiron. Accessed March 2, 2020. https://flatiron.com/ real-world-evidence/

22. National Comprehensive Cancer Network. Genetic/familial high-risk assessment: breast and ovarian. Version 2.2021. National Comprehensive Cancer Network. Accessed October 6, 2020. https://www2.tri-kobe.org/nccn/ guideline/gynecological/english/ genetic familial.pdf

23. Society of Gynecologic Cancer. SGO Clinical practice statement: genetic testing for ovarian cancer (SGO, October 2014). Society of Gynecologic Cancer. Published October 1, 2014. Accessed October 6, 2020. https://www.sgo. org/clinical-practice/guidelines/ genetic-testing-for-ovarian-cancer/
24. Foote JR, Lopez-Acevedo M, Buchanan AH, et al. Cost comparison of genetic testing strategies in women with epithelial ovarian cancer. J Oncol Pract. 2017;13(2):e120-e129. doi:10.1200/ JOP.2016.011866

25. Avastin. Prescribing information. Roche; 2004. Accessed October 6, 2020. https://www.accessdata.fda.gov/drugsatfda docs/label/2011/125085s225lbl.pdf

26. Moore K, Colombo N, Scambia G, et al. Maintenance olaparib in patients with newly diagnosed advanced ovarian cancer. N Engl J Med. 2018;379(26):2495-505. doi:10.1056/NEJMoa1810858

27. GlaxoSmithKline. Data on file: wholesale acquisition costs sourced from the Wolters Kluwer/MDDB price database.

28. Guy H, Walder L, Fisher M. Costeffectiveness of niraparib versus routine surveillance, olaparib and rucaparib for the maintenance treatment of patients with ovarian cancer in the United States. Pharmacoeconomics. 2019;37(3):391-405. doi:10.1007/s40273-018-0745-z

29. American Medical Associations. Medicare coding \& payment for drug administration services under the physician fee schedule. Published 2018.

Accessed October 6, 2020. https://www.ja nssencarepath.com/sites/www.janssen carepath.com/files/medicare-codingand-payment-drug-admin-services-physician-fee-schedule.pdf

30. Ray-Coquard I, Pautier P, Pignata S, et al. Olaparib plus bevacizumab as firstline maintenance in ovarian cancer. $\mathrm{N}$ Engl J Med. 2019;381:2416-28. doi:10.1056/ NEJMoa1911361

31. National Comprehensive Care Network. NCCN Clinical Practice Guidelines in Oncology: ovarian cancer including fallopian tube cancer and primary peritoneal cancer. Version 1.2020. Published March 11, 2020. Accessed December 7, 2020. https://www.nccn. org/professionals/physician gls/pdf/ ovarian.pdf 
32. National Institute for Health and Care Excellence. Final appraisal determination: niraparib for maintenance treatment of relapsed, platinum-sensitive ovarian, fallopian tube and peritoneal cancer. NICE. Published May 2018. Accessed October 6, 2020. https://www.nice. org.uk/guidance/ta528/documents/ final-appraisal-determination-document

33. Briggs AH, Weinstein MC, Fenwick EAL, Karnon J, Sculpher MJ, Paltiel AD; ISPOR-SMDM Modeling Good Research Practices Task Force. Model parameter estimation and uncertainty: a report of the ISPOR-SMDM Modeling Good Research Practices Task Force-6. Value Health. 2012;15(6):835-42. doi:10.1016/j.jval.2012.04.014
34. Neeser K, O'Neil W, Stern L, et al. Budget impact of niraparib as maintenance treatment in recurrent ovarian cancer following platinum-based chemotherapy. J Comp Eff Res. 2019;8(8):577-87. doi:10.2217/cer-2018-0069

35. European Medicines Agency. Avastin: bevacizumab. European Medicines Agency. Published July 24, 2020. Accessed October 6, 2020. https://www.ema. europa.eu/en/medicines/human/EPAR/ avastin
36. Wu L, Zhong L. Budget impact analysis of niraparib and olaparib for maintenance treatment of platinum-sensitive, recurrent ovarian cancer in the US. J Med Econ. 2019;22(2):187-95. doi:10.1080/13696998.2 018.1557199

37. National Cancer Institute. Cancer stat facts: ovarian cancer. SEER. Accessed January 9, 2020. https://seer.cancer.gov/ statfacts/html/ovary.html 